\title{
Social and behavioral interventions for improving quality of life of HIV infected people receiving antiretroviral therapy: a systematic review and meta-analysis
}

\author{
Dharma Nand Bhatta ${ }^{1,2,3^{*}}$, Tippawan Liabsuetrakul ${ }^{2}$ and Edward B. McNeil ${ }^{2}$
}

\begin{abstract}
Background: Improvement in quality of life is crucial for HIV infected people. Social and behavioral interventions have been implemented in different contexts to improve the quality of life among HIV infected people. This review appraises the evidence for available interventions that focused on quality of life of HIV infected people receiving antiretroviral therapy (ART).
\end{abstract}

Methods: We searched electronic databases for randomized controlled trials of interventions to improve the quality of life of HIV infected people receiving ART. We searched PUBMED and the Cochrane Centre Register of Controlled Trials (CENTRAL) with the terms "social", "behavioral", "educational", "quality of life", "HIV", and "RCT". Searches were conducted for articles published from 1980 to December 16, 2015. Standardized data abstraction methods and searching steps were applied.

Results: Twenty-eight studies reported the impact of social or behavioral interventions in quality of life among HIV infected people, of which 15 were conducted in United States of America. A total of 4136 participants were enrolled. Of the 28 studies, four studies included females, two studies included males and remaining studies excluded both males and females. The overall reported methodological quality of the studies was subject to a high risk of bias and the study criteria were unclear in most studies. Twenty-one studies reported a significant intervention effect on at least one quality of life domain. Meta-analyses showed significant improvement in general health, mental health, physical function and environment domains of quality of life among intervention groups. However, the expected impact of the intervention was low to moderate because the rigorousness of the studies was low, information was limited, the sample sizes were small and other the quality of the study designs were poor.

Conclusions: Although the available evidence suggests that existing social and behavioral interventions can improve some quality of life domains, the quality of evidence was insufficient to support the notion that these interventions can improve the overall quality of life of HIV infected people receiving ART. Well-designed and rigorous randomized controlled trials with high methodological quality are required.

Keywords: Social intervention, Behavioural intervention, Randomized, HIV, Quality of life, ART

\footnotetext{
* Correspondence: dnbhatta@yahoo.com

${ }^{1}$ Department of Community Medicine and Public Health, Tribhuvan

University, Peoples' Dental College, Kathmandu, Nepal

${ }^{2}$ Faculty of Medicine, Epidemiology Unit, Prince of Songkla University, Hat

Yai, Thailand

Full list of author information is available at the end of the article
} 


\section{Background}

Globally, at the end of 2015, there were approximately 16 million people receiving antiretroviral therapy (ART) out of 36.9 million HIV infected people [1]. United Nation's agencies developed the 90-90-90 strategy (90\% of all people living with HIV will know their HIV status, $90 \%$ of all people with diagnosed HIV infection will receive sustained ART and $90 \%$ of all people receiving ART will have viral suppression) to be achieved by the year 2020 that will help end HIV epidemics [2]. Revised comprehensive ART guidelines developed by World Health Organization (WHO) [3] aim to help prevent the HIV burden and improve the quality of life of HIV infected people. ART has several public health benefits $[4,5]$ but its side effects can alter adherence and affect quality of life [6]. Environmental, social, structural, and personal factors can also affect adherence to ART and quality of life [7].

The concept of quality of life is: "an individual's perception of their position in life in the context of the culture and value systems in which they live and in relation to their goals, expectations, standards and concerns" [8]. Quality of life may be improved through different coping mechanisms, self-efficacy, social, psychological, structural and environmental adjustments. HIV burden is associated with clinical, psychological, behavioral and social problems. Various studies measured the quality of life which covers psychological, social and behavioral aspect [9-11]. Intervention with revised component into the routine health care services may help to improve the quality of life of HIV infected people.

Behavioral or social interventions are developed based on different theories that changes an individual's psychological and social attributes [12]. Behavioral or social interventions provide the chance to share experiences among groups which would help to reduce the loneliness, negative feelings and stress of HIV infected people [13]. Motivation, social and psychological support enhances empowerment and social support among HIV infected populations to access health care services, improve their quality of life and ability to cope with stigma [14, 15]. Furthermore, involvement in the intervention can improve the rate of retention in care or adherence to ART, reduce risky sexual behaviors and increase negotiation skills, self-efficacy, social networks, and communication skills [10, 16]. Systematically integrated behavioral or social interventions for HIV infected people may help to improve access to health care service, reduce the risk of transmission and improve quality of life.

Several interventions have been developed around the world to improve the quality of life of HIV infected people. Comprehensive systematic reviews and metaanalyses based on interventions that focus on improving quality of life among HIV infected individuals receiving
ART are lacking and existing evidence on the effectiveness of preventive interventions is limited [17]. Systematic reviews of studies found that interventions which covered support groups, social services and combined aerobic and resistance exercises had a low to moderate impact on the quality of life of HIV infected people [18-20]. Evidence based findings are required to prioritize activities and resources and develop strategically planned policies. The aim of this review is to summarize the available evidence for behavioral and social interventions for HIV infected populations in order to provide a direction to funding agencies, policy makers, planners and program developers on how best to use their resources to improve the quality of life of HIV infected people.

\section{Methods}

\section{Search methods for identification of studies}

We searched the literature in MEDLINE/PUBMED and Cochrane Library databases from 1980 to 16 December 2015. We developed a standard protocol for the literature search and used standard Mesh terms for PUBMED and Cochrane Centre Register of Controlled Trials (CENTRAL).

The search strategy focused on three keywords: study participants, design and interventions. The search strategy was as follows:

\author{
\#1 social OR behavioral OR behavioural OR \\ educational \\ \#2 quality of life OR QoL \\ \#3 HIV OR human immunodeficiency virus \\ \#4 drug OR medication OR clinical \\ \#5 RCT OR randomized
}

\section{\#1 AND \#2 AND \#3 NOT \#4 AND \#5}

All the studies were searched by two independent authors. Search process and strategy, search record and retrieved studies were reported and documented as per the PRISMA guidelines. Duplicate citations were checked and removed by importing search results to a reference management software system. Only publications in peerreviewed journals and in English were considered.

\section{Eligibility criteria}

This review included all the randomized controlled trials conducted among HIV infected populations who were aged more than or equal 18 years and receiving ART. Social, behavioral or educational interventions were compared with control groups. The quality of life of $\mathrm{HIV}$ infected people receiving ART was reported to be one of outcome measures in the studies. The outcomes 
must have been compared at baseline and a predefined follow up time period.

\section{Data collection and analysis}

Two authors independently screened the studies returned using the predefined keyword search strategy. Relevant study titles and abstracts were independently evaluated. The full text article was obtained for complete assessment after authors considered eligibility based on the title and abstract. Full text of the articles was reviewed by two reviewers independently to assess the inclusion criteria. Final selection of the articles was made by agreement between the two reviewers.

Information abstracted from each study included authors, year published, country, study design, settings, sample size, characteristics of participants, theory used, eligibility criteria, comparison group intervention, intervention components, intervention methods and duration, measurement tools and outcomes. Retention rate in the intervention group, missing data and follow up period were also extracted. The data, independently extracted by two reviewers, were compared and a consensus was made after discussion of discrepancies. The study authors were contacted to obtain clarification of missing or insufficient data.

\section{Assessment of risk of bias in included studies}

Cochrane Handbook for Systematic Reviews of Interventions [21] guidelines were used to assess the risk of bias in all studies included in the review. Assessment criteria included random sequence generation, allocation concealment, incomplete outcomes reporting of sources of bias, blinding of participants, researcher or outcome assessors, completeness of outcome data, selectivity of outcome reporting, missing data and retention rate. The two review authors rated the risk of bias by assessing either "yes" (low risk of bias), "no" (high risk of bias) or "unclear" (insufficient information) to all probable sources of bias. Criteria for rating internal and external validity (good, fair, poor), quality of evidence for each individual study (Table 1, rated as strong, medium, weak), overall quality of the body of evidence by outcome of interest (Table 2, rated as good, fair, poor) and expected impact of the intervention on the outcome of interest (Table 3, rated as high, moderate, low, uncertain) were adapted from the United States Preventive Services Task Force procedure manual [22, 23].

\section{Statistical assessment}

Pooled effects were obtained by calculating standardized mean differences with $95 \%$ confidence intervals. Analysis was done using both fixed-effects and random-effects models. When there was more than one intervention arm, the mean and standard deviation was combined using Review Manager Calculator. We compared the
Table 1 Criteria for rating the quality of evidence for individual studies

\begin{tabular}{ll}
\hline Level of Evidence & Description \\
\hline $1=$ Strong & $\begin{array}{l}\text { Systematic review/meta-analysis of RCTs with } \\
\text { consistent findings; high-quality individual RCT }\end{array}$ \\
& $\begin{array}{l}\text { Systematic review/meta-analysis of lower-quality } \\
\text { clinical trials or of studies with inconsistent }\end{array}$ \\
& $\begin{array}{l}\text { findings; lower quality clinical trial; cohort study; } \\
\text { case-control study } \\
3=\text { Weak }\end{array}$ \\
& $\begin{array}{l}\text { Consensus guidelines; usual practice; expert } \\
\text { opinion; case series }\end{array}$ \\
\hline
\end{tabular}

social and behavioral intervention versus a control group. Statistical heterogeneity of the reviewed studies was assessed using Cochran's Q-test, quantified using $\mathrm{I}^{2}$ and categorized as low $(0-25 \%)$, moderate $(26-50 \%)$ or high $(>50 \%)$ [21]. We performed subgroup analyses to eliminate the heterogeneity based on the follow up duration. Sensitivity analyses were performed if high heterogeneity was detected. This meta-analysis was performed using Review Manager Software Version 5.3.

The protocol was approved by the human research Ethics Committee, Faculty of Medicine, Prince of Songkla University, Thailand (REC Number: 59-146-18-1).

\section{Results}

\section{Study selection}

A total of 167 publications were identified from the electronic databases using the search strategy. After excluding duplicate publications, 156 articles remained (Fig. 1). Of these, 104 did not meet the inclusion criteria. Of the 52 remaining publications, 24 were excluded: ten did not report clear information about ART medication, four were not randomized clinical trials (RCTs), three used a

Table $\mathbf{2}$ Criteria for rating the overall quality of the body of evidence by outcome of interest

\begin{tabular}{|c|c|}
\hline Rating & Description \\
\hline $1=\mathrm{Good}$ & $\begin{array}{l}\text { Evidence includes consistent results from } \\
\text { well-designed well-conducted studies in } \\
\text { representative populations that directly } \\
\text { assess effects on health outcomes }\end{array}$ \\
\hline $2=$ Fair & $\begin{array}{l}\text { Evidence is sufficient to determine effects } \\
\text { on health outcomes, but the strength of } \\
\text { the evidence is limited by the number, } \\
\text { quality, or consistency of the individual } \\
\text { studies, generalizability to routine practice, } \\
\text { or indirect nature of the evidence on } \\
\text { health outcomes }\end{array}$ \\
\hline $3=$ Poor & $\begin{array}{l}\text { Evidence is based on consensus, usual } \\
\text { practice, opinion, or case series. Additionally } \\
\text { evidence is insufficient to fully assess the } \\
\text { effects on health outcomes because of limited } \\
\text { number, or power of studies, important flaws } \\
\text { in design or conduct, gaps in the chain of } \\
\text { evidence, or lack of information on importance } \\
\text { on the key health outcomes }\end{array}$ \\
\hline
\end{tabular}


Table 3 Criteria for rating the expected impact of the intervention on the outcome of interest

\begin{tabular}{ll}
\hline Grade & Definition \\
\hline High & $\begin{array}{l}\text { We are very confident that the estimate of effect lies } \\
\text { close to the true effect for this outcome. The body } \\
\text { of evidence has few or no deficiencies. We believe } \\
\text { that the findings are stable (i.e., another study would } \\
\text { not change the conclusions). }\end{array}$ \\
Moderate $\quad \begin{array}{l}\text { We are moderately confident that the estimate of effect } \\
\text { lies close to the true effect for this outcome. The body } \\
\text { of evidence has some deficiencies. We believe that the } \\
\text { findings are likely to be stable, but some doubt remains. }\end{array}$ \\
$\begin{array}{l}\text { We have limited confidence that the estimate of effect } \\
\text { lies close to the true effect for this outcome. The body } \\
\text { of evidence has major or numerous deficiencies (or both). } \\
\text { We believe that additional evidence is needed before } \\
\text { concluding either that the findings are stable or that } \\
\text { the estimate of effect is close to the true effect. } \\
\text { We have no evidence, we are unable to estimate an } \\
\text { Insufficient } \\
\text { effect, or we have no confidence in the estimate of } \\
\text { the body of evidence has unacceptable deficiencies, } \\
\text { precluding us from reaching a conclusion. }\end{array}$ \\
\hline
\end{tabular}

medical intervention, three had not quality of life outcome, one had not social or behavioral intervention, one was conducted in a pediatric population, one included participants from 16 years of age, and one included nonHIV participants. Finally, 28 publications were included in this review.

\section{Study characteristics}

Table 4 presents the characteristics of the 28 studies [24-51]. All studies were RCTs and published from 2002 to 2014. Studies were conducted in the following countries: United States of America (15), Canada (1), Switzerland (1), France (1), Spain (1), Brazil (1), Rwanda (1), South Africa (1), Australia (1), China (2), Vietnam (1), Thailand (1), and multicenter settings (1: South Africa, Puerto Rico, USA). Study duration ranged from 6 to 54 months and six studies did not mention the duration of study. The number of participants per study ranged from 22 to 507 .

\section{Intervention characteristics}

All 28 studies applied different types of interventions. Control groups for 14 of the studies received standard care (standard or wait-list control or comparison group), and in the remaining studies included group exercise (1), standard routine care and symptom management manual (2), healthy eating education (1), nutritional support and care (1), unsupervised walking program and monthly group forum (1), therapist guided exercise (1), individual psycho-educational condition (1), heat therapy and reading magazine (1), no exercise (1), counseling (1), treatment by peers (1), individual psychotherapy (1), and adherence counseling (1). The studies had a wide variety of intervention sessions and types of interventionists.

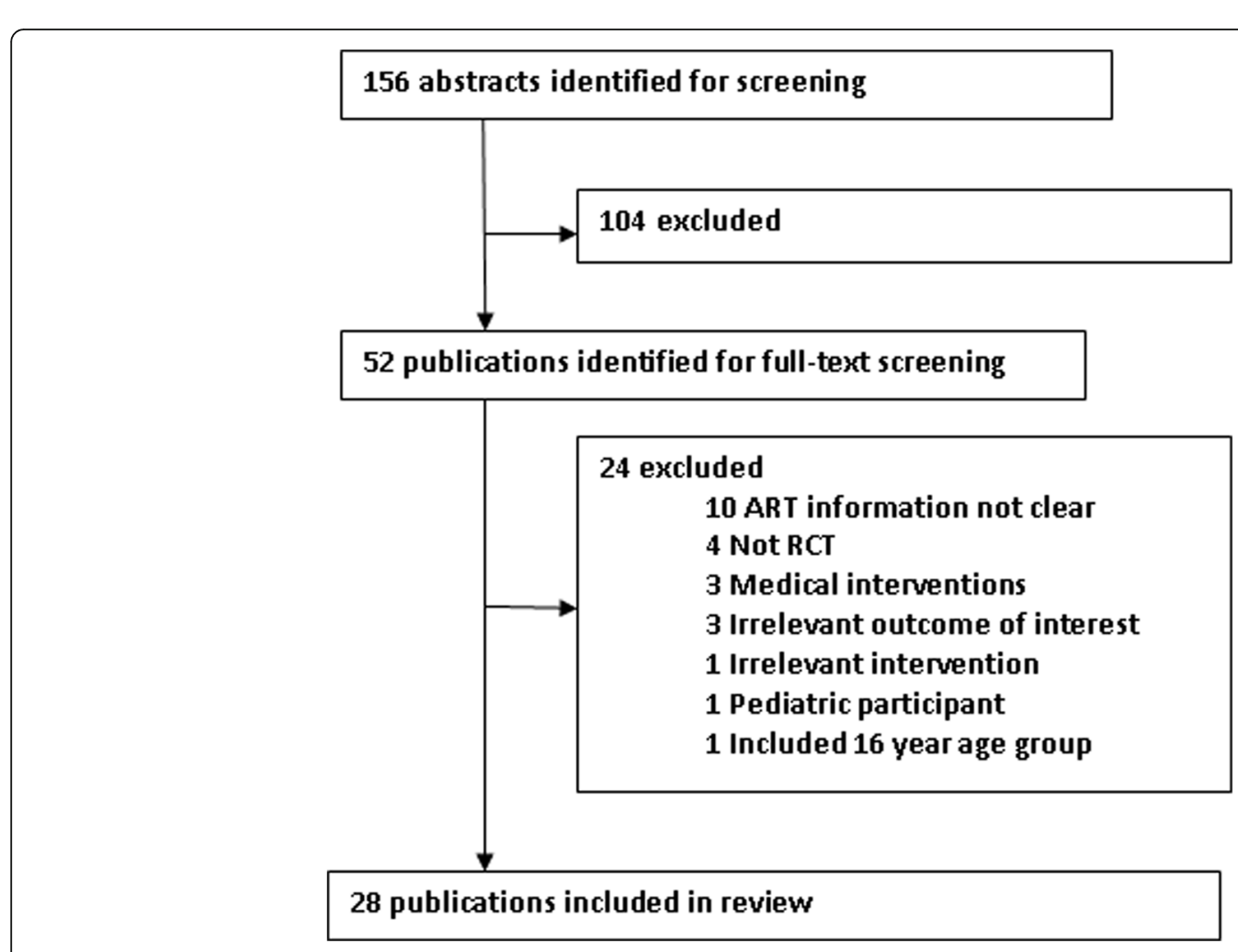

Fig. 1 Study selection flowchart 


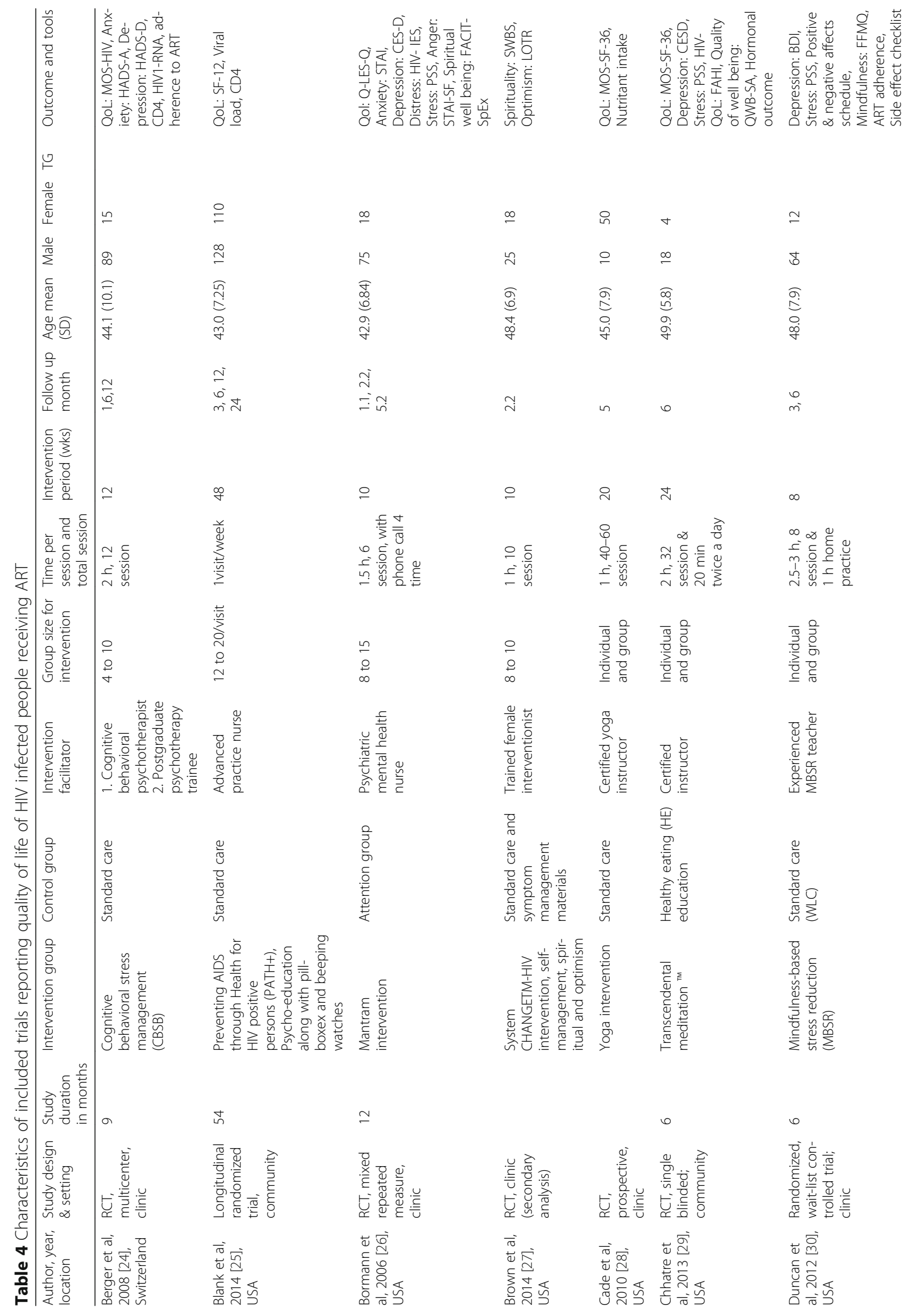




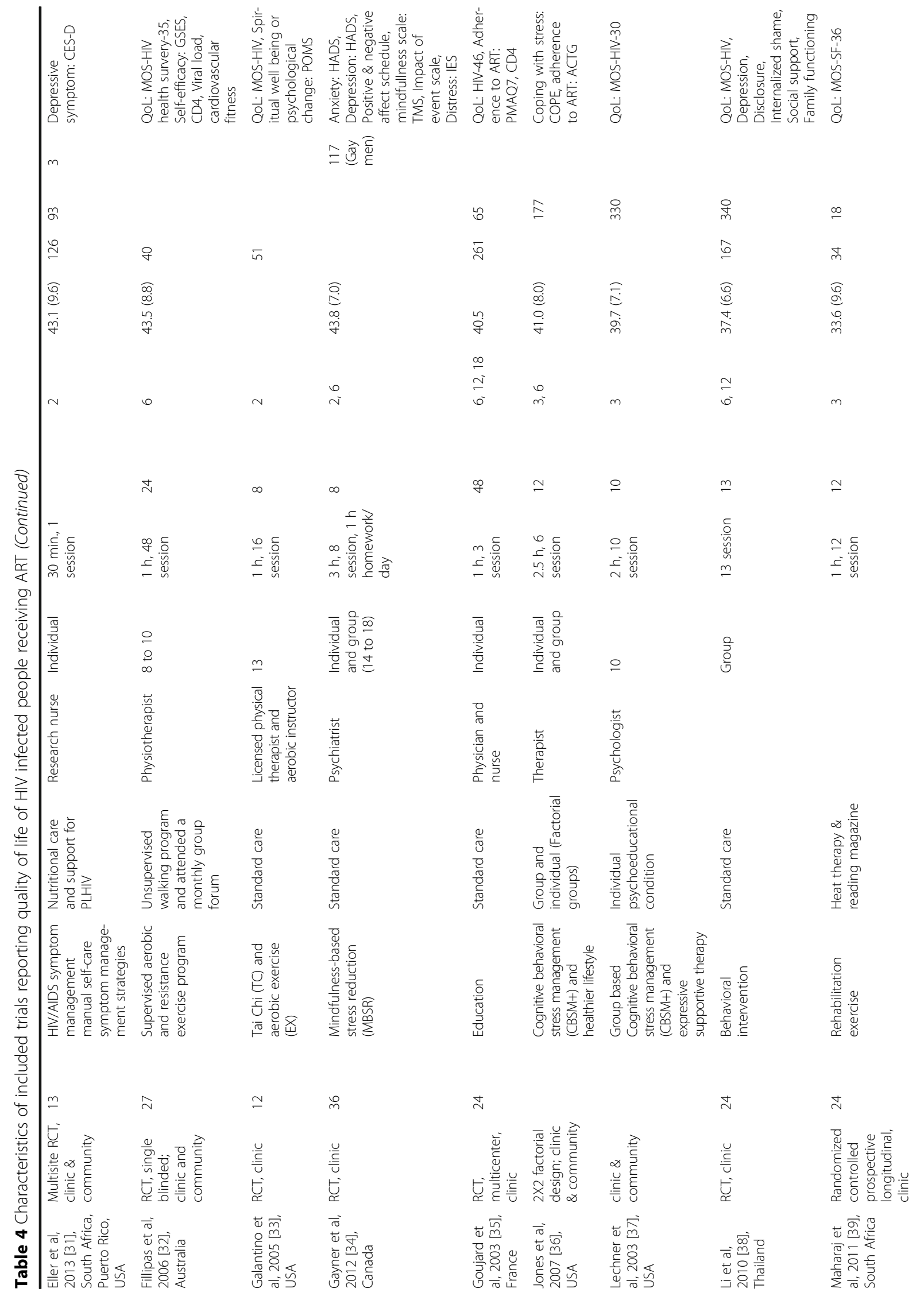




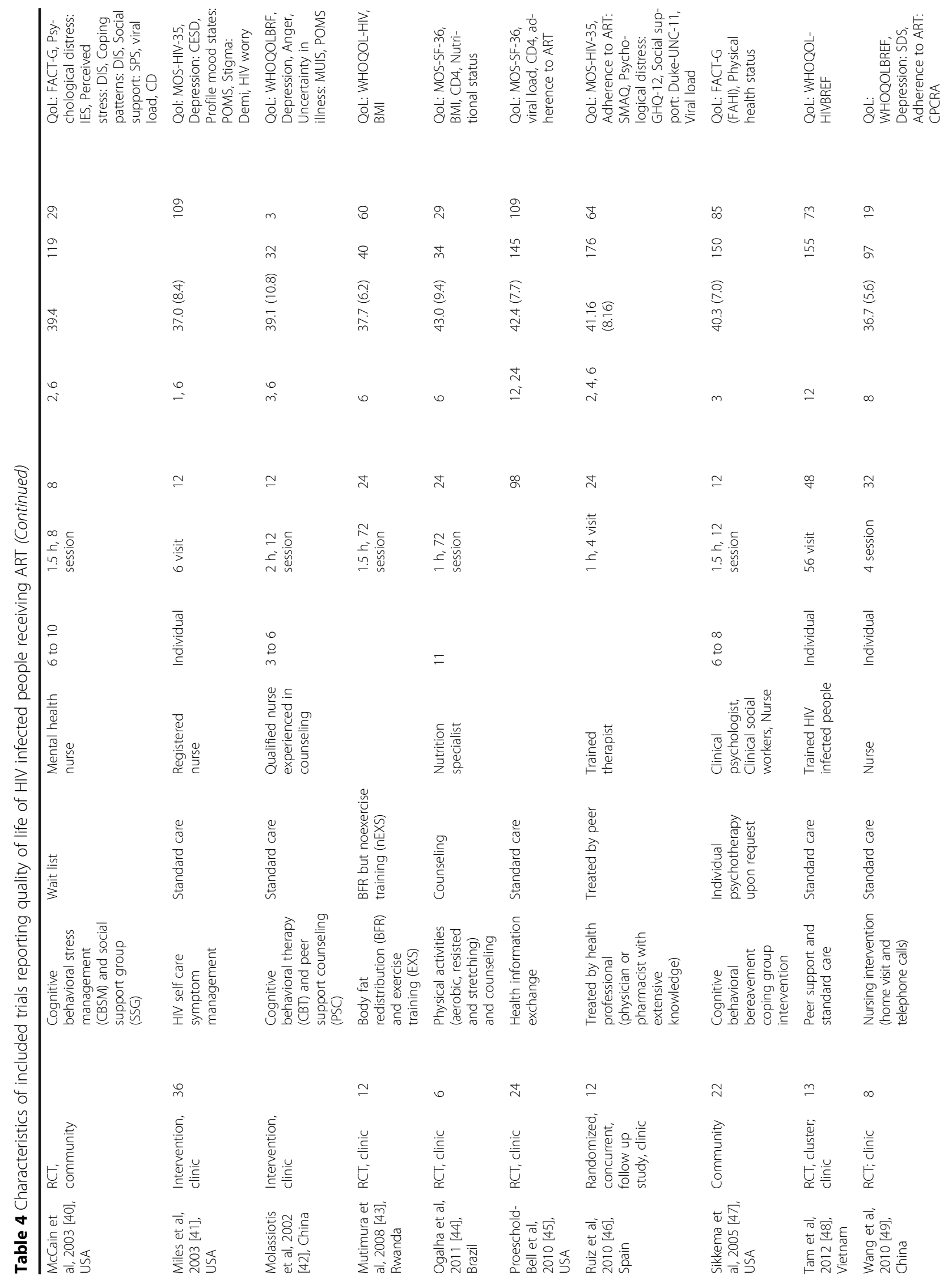




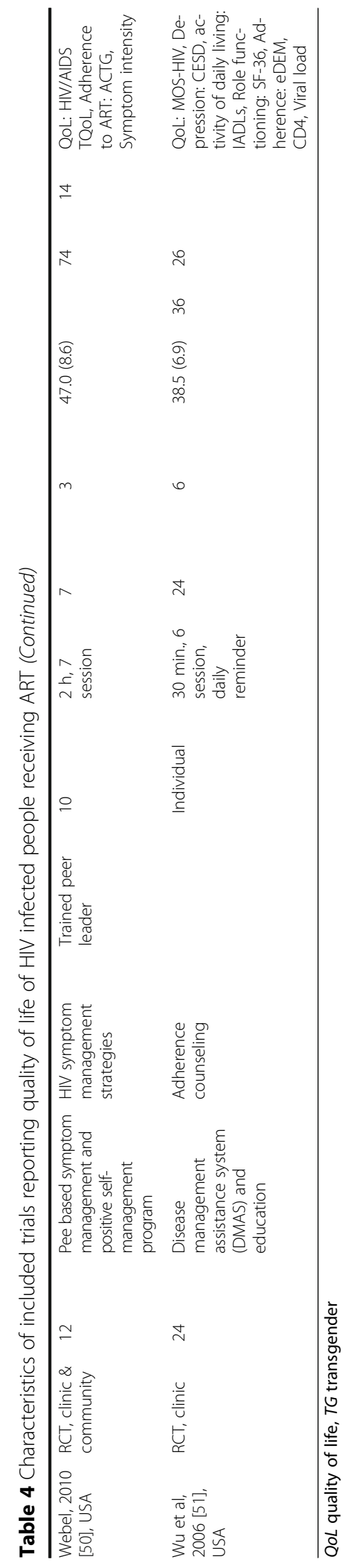




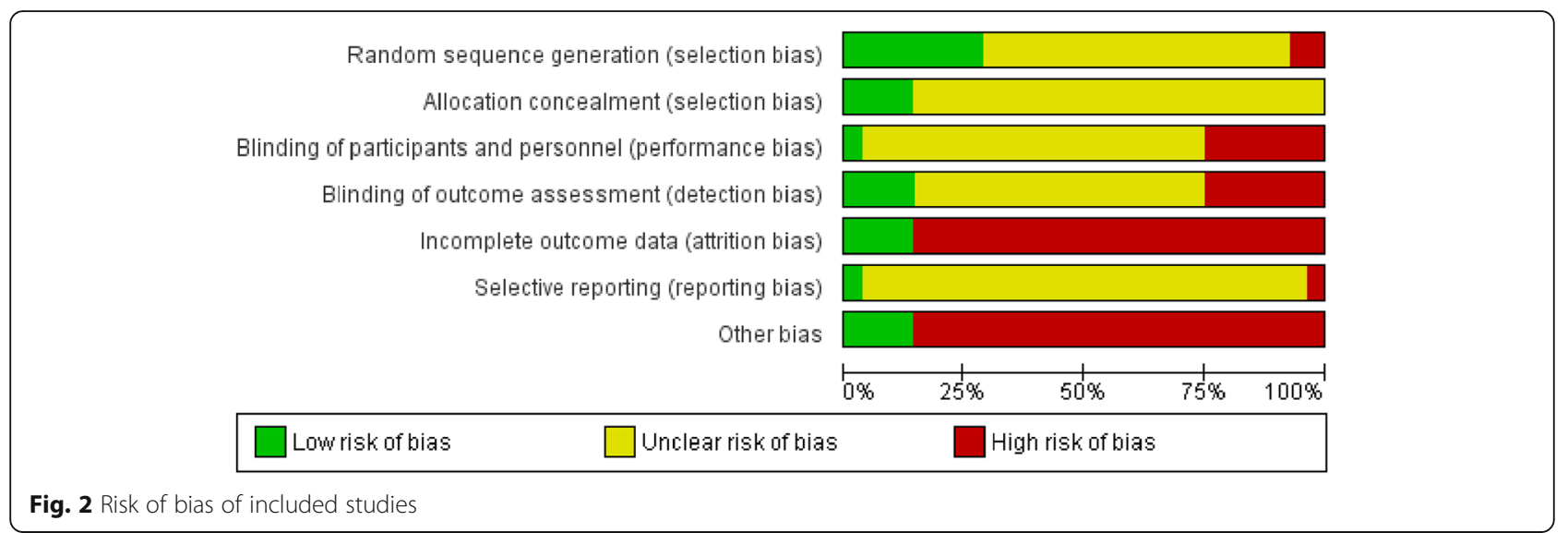

The intervention period ranged from 7 to 98 weeks and the follow up period ranged from 1 to 24 months. The mean age for all study participants ranged from 33.6 to 49.9 years. Four studies included only females [36, 37, 41, $50]$, and two included only males [32, 33] (Table 4).

\section{Study quality or risk of bias}

Cochrane risk of bias tool was used to determine the quality of methods among all 28 studies (Fig. 2). Eight studies reported details of random sequence generation methods $[24,26,30,32-34,41,51]$ and seven studies provided details of their allocation concealment [24, 26, 30, 32, 42, $45,46]$. Ten studies provided information related to participant and personnel or outcome assessment blinding [24-26, 29, 32-34, 37, 39, 46]. Eleven studies did not mention the reasons for participants' withdrawals $[25,27$, $29,35,39-41,43,44,50,51]$ and 13 studies described an intention-to-treat analysis approach $[24,26,27,29,30$, $32-34,38,41,46,47,50]$. Table 5 shows the key quality of evidence and impact of the individual study.

\section{Quality of life outcome}

All studies reported that quality of life was one of the study outcomes. Eight studies mentioned quality of life as the primary outcome $[26,33,45,47-51]$, three studies mentioned quality of life as the secondary outcome $[24,32,46]$ and 17 studies did not provide the information that quality of life was measured either primary or secondary outcome. Quality of life was measured using different tools: MOS-HIV health survey (MOS-HIV or MOS-HIV-35 or MOS-HIV-30) [24, 32, 33, 37, 38, 41, $46,51]$, SF-36 health survey [28, 29, 39, 44, 45], SF-12 health survey [25], quality of life enjoyment and satisfaction questionnaire (Q-LES-Q) [26], HIV-46 [35], functional assessment of cancer therapy (FACT-G) [40, 47], WHOQOLBREF [42, 49], WHOQOL-HIV [43, 48], HIV/AIDS targeted quality of life instrument [50], positive and negative affects schedule (PANAS) [30, 34], and spiritual well-being scale (SWBS) [27]. Different
Table 5 Validity, quality and impact of the individual study

\begin{tabular}{|c|c|c|c|}
\hline ID & $\begin{array}{l}\text { Internal } \\
\text { validity }\end{array}$ & $\begin{array}{l}\text { External } \\
\text { validity }\end{array}$ & $\begin{array}{l}\text { Quality of evidence } \\
\text { for individual studies }\end{array}$ \\
\hline Berger et al, 2008 [24] & Fair & Fair & Medium \\
\hline Blank et al, 2014 [25] & Poor & Fair & Medium \\
\hline Bormann et al, 2006 [26] & Poor & Fair & Medium \\
\hline Brown et al, 2014 [27] & Fair & Fair & Medium \\
\hline Cade et al, 2010 [28] & Poor & Poor & Weak \\
\hline Chhatre et al, 2013 [29] & Fair & Poor & Weak \\
\hline Duncan et al, 2012 [30] & Fair & Poor & Medium \\
\hline Eller et al, 2013 [31] & Poor & Poor & Weak \\
\hline Fillipas et al, 2006 [32] & Fair & Fair & Medium \\
\hline Galantino et al, 2005 [33] & Poor & Poor & Medium \\
\hline Gayner et al, 2012 [34] & Fair & Fair & Medium \\
\hline Goujard et al, 2003 [35] & Poor & Poor & Medium \\
\hline Jones et al, 2007 [36] & Poor & Poor & Medium \\
\hline Lechner et al, 2003 [37] & Poor & Poor & Medium \\
\hline Li et al, 2010 [38] & Fair & Good & Strong \\
\hline Maharaj et al, 2011 [39] & Poor & Poor & Weak \\
\hline McCain et al, 2003 [40] & Poor & Poor & Medium \\
\hline Miles et al, 2003 [41] & Poor & Poor & Medium \\
\hline Molassiotis et al, 2002 [42] & Poor & Fair & Medium \\
\hline Mutimura et al, 2008 [43] & Fair & Poor & Medium \\
\hline Ogalha et al, 2011 [44] & Poor & Poor & Medium \\
\hline Proeschold-Bell et al, 2010 [45] & Poor & Poor & Medium \\
\hline Ruiz et al, 2010 [46] & Fair & Fair & Medium \\
\hline Sikkema et al, 2005 [47] & Fair & Good & Medium \\
\hline Tam et al, 2012 [48] & Fair & Fair & Medium \\
\hline Wang et al, 2010 [49] & Poor & Poor & Weak \\
\hline Webel, 2010 [50] & Fair & Poor & Medium \\
\hline Wu et al, 2006 [51] & Poor & Fair & Medium \\
\hline
\end{tabular}




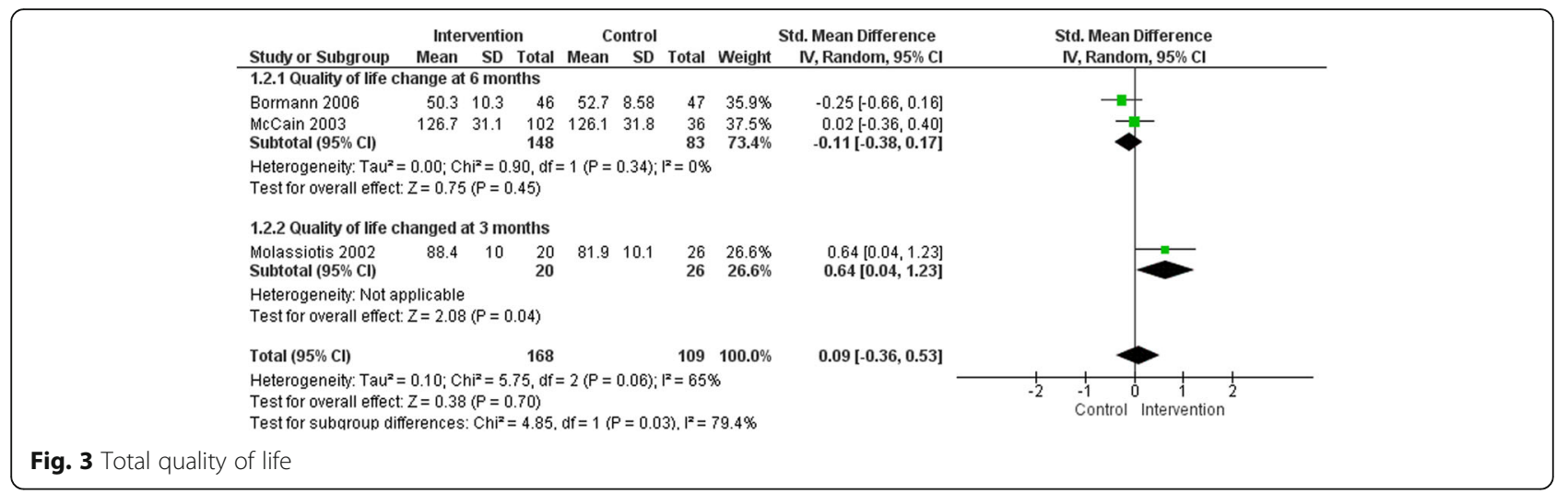

studies used different instruments to assess quality of life and different instruments cover different dimensions of quality of life. Seven studies reported no intervention effect on any quality of life domain, of which one reported only one domain [26], two reported four domains [31,35], one reported 10 domains [45], one reported two domains [46], one reported seven domains [50], and one study reported 11 domains [51] in their final results.

Twenty-one studies reported a better improvement in quality of life scores among the intervention group

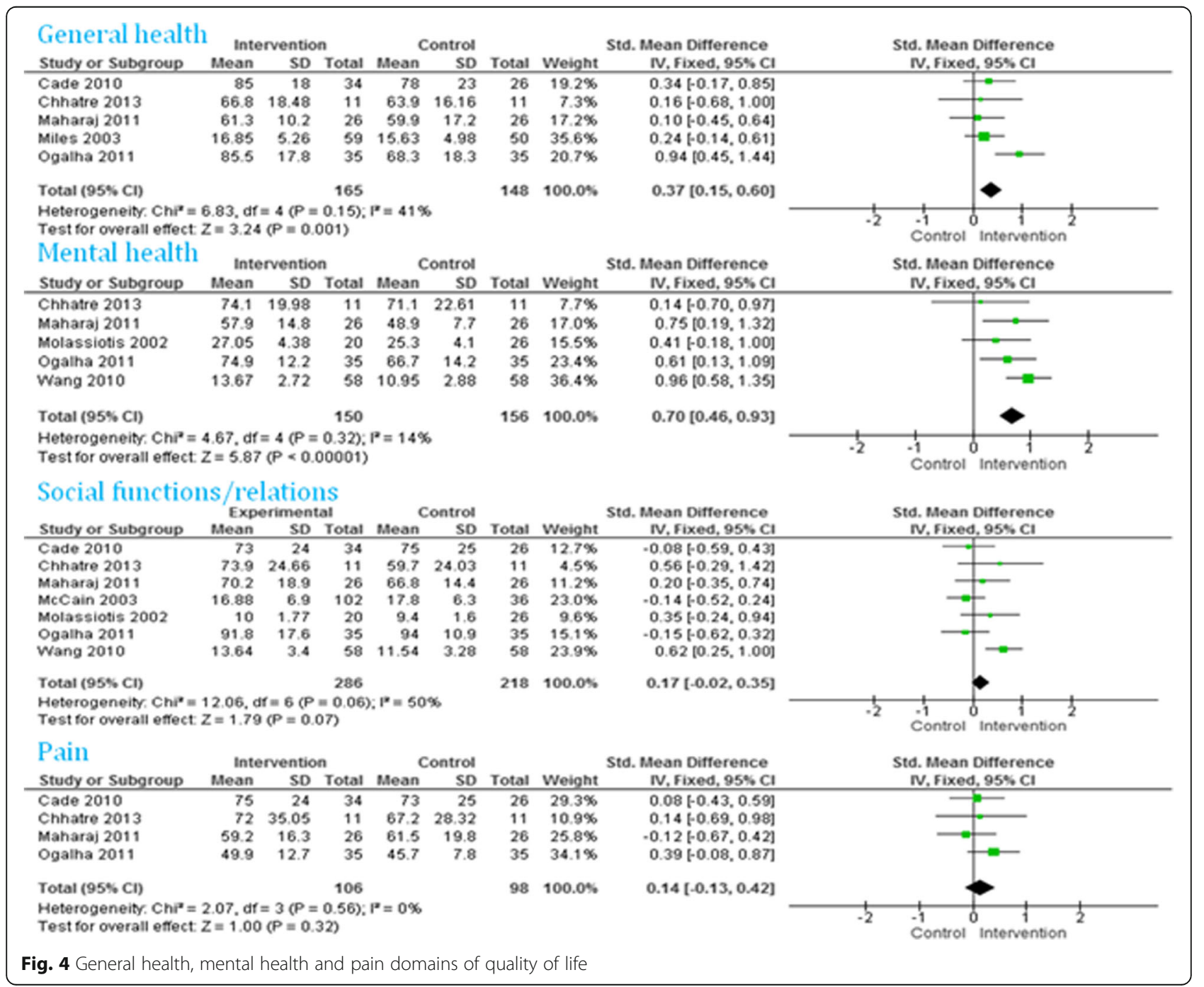


compared to the control group. Of these, six studies reported an improvement in all the domains: two out of two domains [24, 25, 27, 40], three out of three domains [48], and four out of four domains [49]. The remaining studies reported that four out of five [41, 43], five out of nine [28], five out of eight [29], seven out of 10 [32], four out of 11 [37], two out of three [38], nine out of 10 [39], two out of four [42], six out of eight [44], and four out of six [47], domains improved among the intervention group. The meta-analyses did not find any overall significant intervention effect in total quality of life (Fig. 3), social function, pain (Fig. 4), energy/fatigue, role emotional, emotional well-being (Fig. 5) and role physical (Fig. 6) domains of quality of life. Significant improvements were found in general health (overall effect: $0.37,95 \%$ confidence interval $0.15,0.60)$, mental health (0.70; 0.46, 0.93, Fig. 4), environment (0.76; 0.44, 1.08, Fig. 5) and physical function (0.58; 0.24, 0.91, Fig. 6) domains of quality of life among the intervention group.

\section{Safety}

Only two studies recorded adverse events and no trial reported fidelity of the intervention. A total of 921 participants were lost to follow up or dropped out from the study. Most of the studies did not report the reason for dropping out. Of those, the main reasons were health issues, unavailability of time, emotional issues, no interest, and change of address.

\section{Discussion}

Twenty eight studies included in this review evaluated the impact of social or behavioral interventions on quality of life among HIV infected people with ART. The effects of these interventions should be interpreted with caution since the methodological quality of the studies included in this review was low. Previous systematic reviews highlighted similar findings with methodological issues $[18,19]$.

In our review, apart from six studies which found improvement in quality of life domains, the expected impact

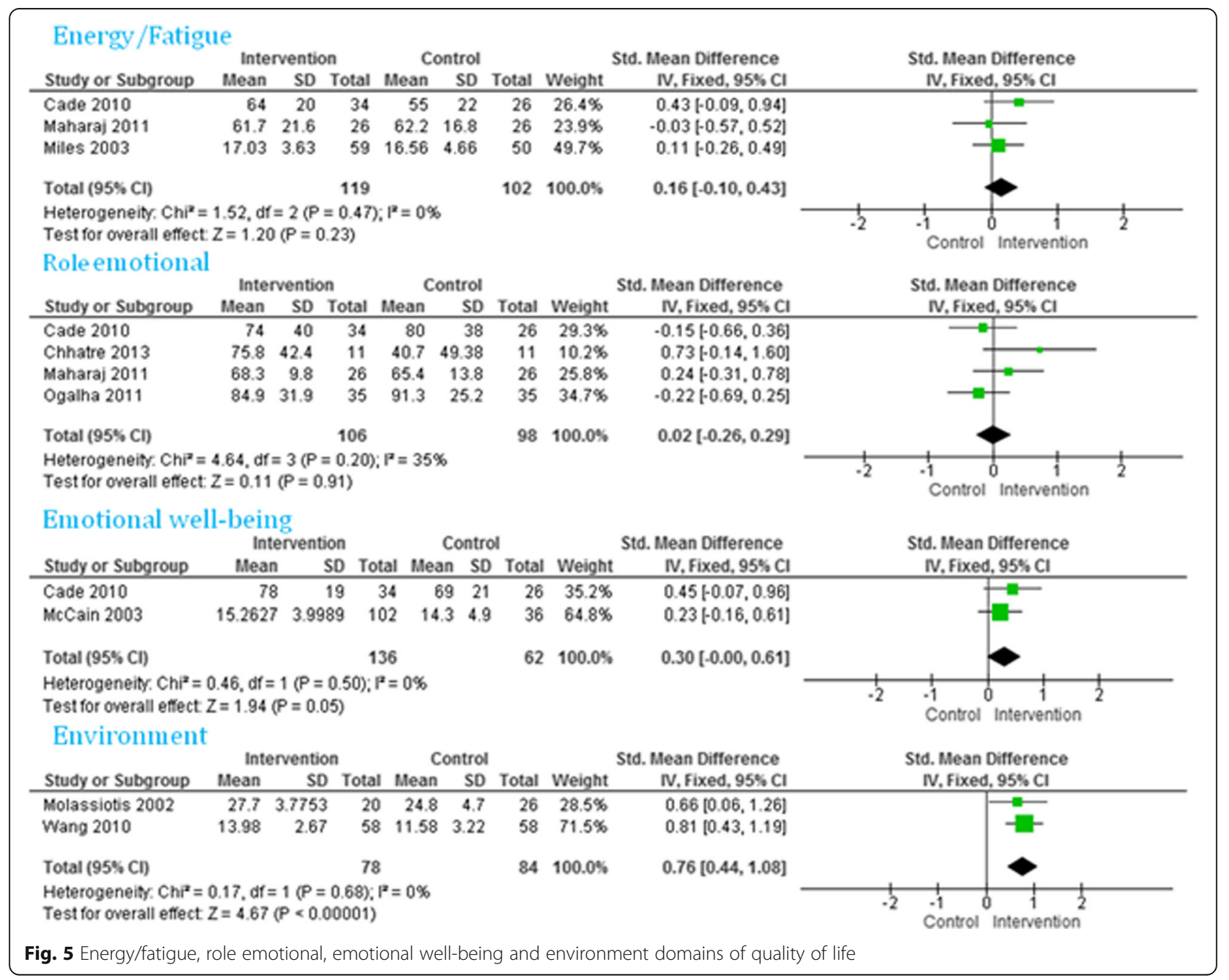




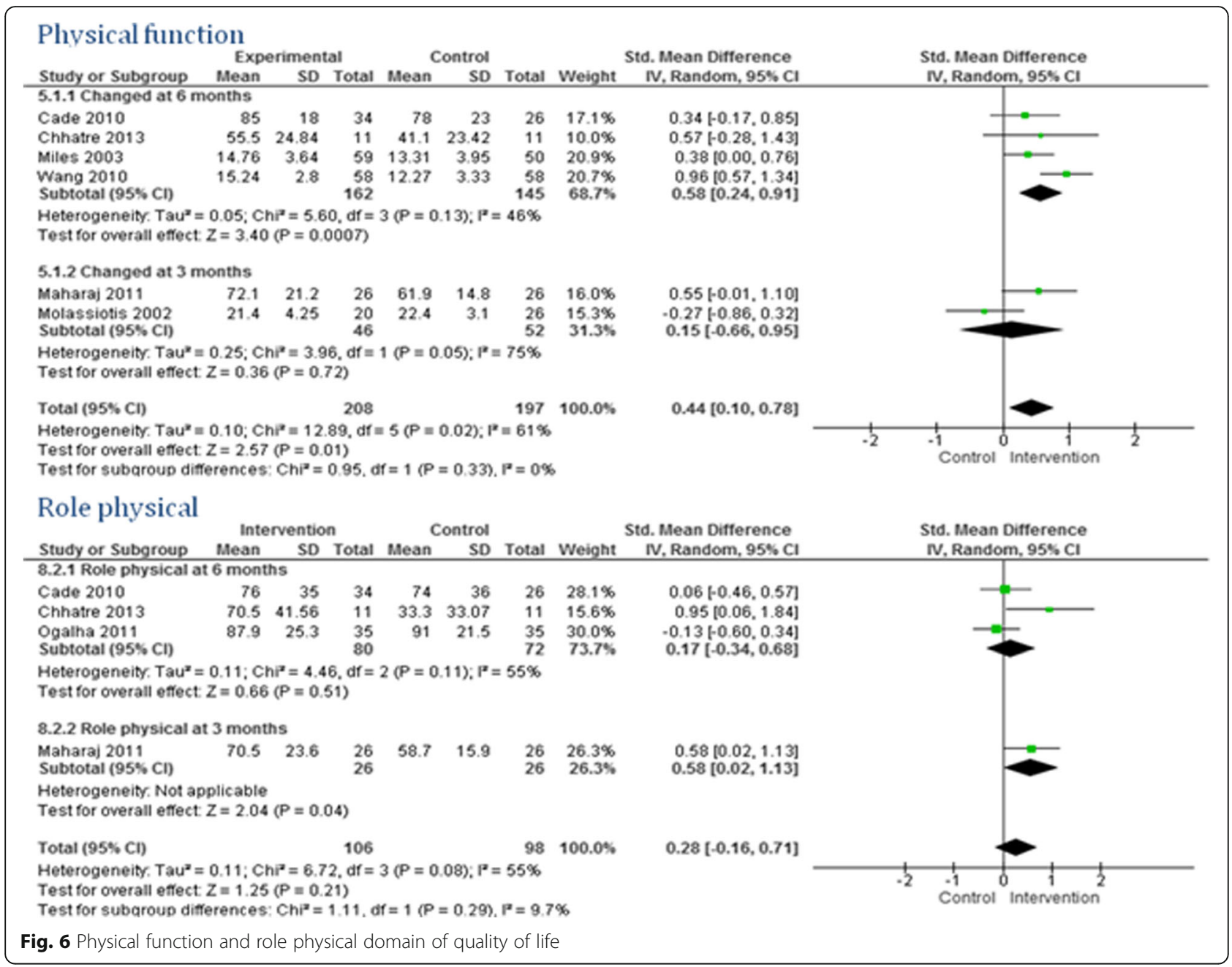

of the intervention was rated as low or moderate based on the available evidence. A previous systematic review highlighted similar findings that evidence was limited to assess the impact of intervention on quality of life [17]. Our findings from the meta-analyses indicated a significant improvement in general health, mental health and physical health domains of quality of life. A previous meta-analysis based on exercise intervention revealed similar findings in that few domains of quality of life were significantly improved by the intervention [20]. Lack of rigorous methods of individual studies and unavailability of sufficient information about recruitment process, blinding, effect size estimation, drop out, and intention to treat, resulted in the expected impact on the outcome being inconclusive.

\section{Applicability of evidence/programmatic considerations for implementation}

Social and behavioral interventions for HIV infected people may improve quality of life along with ART. Intervention effects could be improved through establishment of trustable and sustainable networks with available clinical services and community referral systems. Reliable networking can increase motivational and emotional support to HIV infected people. Implementation of social and behavioral services for HIV infected people is still a challenge.

\section{Research gaps}

There was insufficient evidence to strongly recommend the social and behavioral interventions into clinical practices for improving quality of life among HIV infected people for the following reasons. First, the efficacy of the available social and behavioral interventions is not clear. Therefore rigorous and well-designed studies with large sample sizes to address potential confounding, and longterm follow up are needed. Second, sustainability of the intervention is unclear. Research should address the acceptability, feasibility, applicability and sustainability of interventions. Interventions should be cost-effective and appropriate for local cultures and contexts. Third, most of the studies did not mention validation of quality of 
life measurement tools. Accuracy of the measurement tools was also unclear. Fourth, evidence is not sufficient for targeting sub-groups of HIV populations. More research is needed to formulate HIV prevention and control strategies and policies. In addition, research in this area is needed to develop empowerment and advocacy that play a role in preventing HIV transmission and treatment discontinuation and unavailability. This may have a direct and indirect impact on quality of life of HIV infected people.

\section{Limitations of the current review}

The ability to draw conclusions regarding the effectiveness of social and behavioral interventions among HIV infected people receiving ART is complex. We searched the studies those were written in English language which could limit the sufficient evidences. The interventions in this review were diverse in terms of target populations, type of interventions, delivery persons, measurement tools, duration of intervention and follow up and study duration. The lack of standard measurement tools limits the capacity to evaluate results from individual studies and make conclusions concerning the strength of the evidence. Furthermore, low sample sizes with low statistical power and lack of rigorous methodologies limit the impact and generalizability of the results. Interventions need to have clear guidelines on delivery, method, place and the persons delivering them. All the interventions should be cost-effective and future research should address this component.

\section{Conclusions}

This review has summarized existing evidence on the effect of social and behavioral interventions in improving the quality of life of HIV infected people receiving ART. Based on our review of available evidence and review criterion, social and behavioral interventions are likely to have a low or moderate impact on quality of life. However the methodological limitations can affect the quality of evidence from included studies. Novel and rigorously designed studies and program monitoring and evaluations on HIV outcomes are needed to evaluate the impact of these interventions on key outcomes for quality of life of HIV infected people.

\section{Acknowledgements}

Not applicable.

Funding

No financial support was used for this research.

\section{Availability of data and materials}

All data supporting our findings is contained within the manuscript and the authors can be contacted at dnbhatta@yahoo.com (DNB) for further clarification if required.

\section{Authors' contributions}

DNB and TL searched the literature, conceived and designed the study, acquired and interpreted the data and drafted the manuscript. EM interpreted the data and helped to revise the final manuscript. All authors read and approved the final manuscript.

\section{Authors' information}

Not applicable.

\section{Competing interest}

The authors declare that they have no competing interests.

\section{Consent for publication}

Not applicable.

\section{Ethics approval and consent to participate}

This project was approved by the human research Ethics Committee, Faculty of Medicine, Prince of Songkla University, Thailand (REC Number: 59-146-18-1). This study is based on the review of published articles. All the reviewed and included study had followed the ethical considerations.

\section{Publisher's Note}

Springer Nature remains neutral with regard to jurisdictional claims in published maps and institutional affiliations.

\section{Author details}

${ }^{1}$ Department of Community Medicine and Public Health, Tribhuvan University, Peoples' Dental College, Kathmandu, Nepal. 'Faculty of Medicine, Epidemiology Unit, Prince of Songkla University, Hat Yai, Thailand.

${ }^{3}$ Department of Public Health, Pokhara University, Nobel College, Kathmandu, Nepal.

Received: 3 December 2016 Accepted: 21 April 2017

Published online: 24 April 2017

\section{References}

1. UNAIDS. Fact sheet 2015: global statistic. Geneva, Switzerland: Joint United Nations Programme on HIV/AIDS (UNAIDS); 2015. Accessed 12 Jan 2016. http://www.unaids.org/sites/default/files/media_asset/20150901_FactSheet_ 2015 en.pdf.

2. UNAIDS. The Joint United Nations Programme on HIV/AIDS, 90-90-90 an ambitious treatment target to help end the AIDS epidemic. 2014. p. JC2684

3. WHO. Guideline on when to start antiretroviral therapy and pre-exposure prophylaxis for HIV. Geneva, Switzerland: World Health Organization (WHO); 2015.

4. Braitstein P, Brinkhof $M$, Dabis F, Schechter M, Boulle A, Miotti $P$, et al. Mortality of HIV-1-infected patients in the first year of antiretroviral therapy: comparison between low-income and high-income countries. Lancet. 2006;367:817-24.

5. Fauci AS, Marston HD. Achieving an AIDS-free world: science and implementation. Cell. 2013;155:733-4.

6. Mills EJ, Lester R, Ford N. Promoting long term adherence to antiretroviral treatment. BMJ. 2012;344:e4173.

7. Langebeek N, Gisolf EH, Reiss P, Vervoort SC, Thóra B, Richter C, et al. Predictors and correlates of adherence to combination antiretroviral therapy (CART) for chronic HIV infection: a meta-analysis. BMC Med. 2014;12:142.

8. WHOQOLGroup. Field trial WHOQOL-100, February 1995: facet definitions and questions. Geneva: WHO; 1995 (MNH/PSF/95.1. B).

9. Brown JL, Vanable PA. Cognitive-behavioral stress management interventions for persons living with HIV: a review and critique of the iterature. Ann Behav Med. 2008;35:26-40.

10. Fisher JD, Smith L. Secondary prevention of HIV infection: the current state of prevention for positives. Curr Opin HIV AIDS. 2009;4(4):279-87.

11. Rotheram-Borus MJ, Stein JA, Jiraphongsa C, Khumtong S, Lee S-J, Li L. Benefits of family and social relationships for Thai parents living with HIV. Prev Sci. 2010;11:298-307.

12. Albarracín D, Gillette JC, Earl AN, Glasman LR, Durantini MR, Ho M-H. A test of major assumptions about behavior change: a comprehensive look at the effects of passive and active HIV-prevention interventions since the beginning of the epidemic. Psychol Bull. 2005;131(6):856-97. 
13. Walker J. Rural women with HIV and AIDS: perceptions of service accessibility, psychosocial, and mental health counseling needs. J Ment Health Couns. 2002;24(4):299-316.

14. Wouters E, Van Damme W, van Rensburg D, Masquillier C, Meulemans H. Impact of community-based support services on antiretroviral treatment programme delivery and outcomes in resource-limited countries: a synthetic review. BMC Health Serv Res. 2012;12:194.

15. Qiao S, Li X, Stanton B. Social support and HIV-related risk behaviors: a systematic review of the global literature. AIDS Behav. 2014;18:419-41.

16. Hosek S, Brothers J. Lemos, the Adolescent Medicine Trials Network for HIV/ AIDS Interventions D. What HIV-positive young women want from behaviora interventions: A qualitative approach. AIDS Patient Care STDs. 2012;26:291-7.

17. Medley A, Bachanas P, Grillo M, Hasen N, Amanyeiwe U. Integrating prevention interventions for people living with HIV into care and treatment programs: a systematic review of the evidence. J Acquir Immune Defic Syndr. 2015;68:S286-96.

18. Bateganya $M H$, Amanyeiwe $U$, Roxo $U$, Dong M. Impact of support groups for people living with HIV on clinical outcomes: a systematic review of the literature. J Acquir Immune Defic Syndr. 2015;68:S368-74.

19. Bateganya $\mathrm{MH}$, Dong M, Oguntomilade J, Suraratdecha C. The impact of social services interventions in developing countries: a review of the evidence of impact on clinical outcomes in people living with HIV. J Acquir Immune Defic Syndr. 2015;68:S357-67.

20. Neto MG, Conceição CS, Carvalho VO, Brites C. Effects of combined aerobic and resistance exercise on exercise capacity, muscle strength and quality of life in HIV-infected patients: a systematic review and meta-analysis. PLoS One. 2015;10:e0138066

21. Higgins JP, Green S. The Cochrane Collaboration. Cochrane handbook for systematic reviews of interventions: Cochrane Book Series. Wiley Online Library. Chichester: John Wiley \& Sons Ltd; 2008.

22. Kaplan JE, Hamm TE, Forhan S, Hassani AS, Bang G, Weyant E, et al. The impact of HIV care and support interventions on key outcomes in low-and middle-income countries: a literature review-introduction. J Acquir Immune Defic Syndr. 2015;68:S253-6.

23. US. U.S. Preventive services task force procedure manual. AHRQ publication No. 08-05118-EF 2008, http://www.uspreventiveservicestaskforce.org/Page/ Name/methods-and-processes. Accessed 10 Mar 2016.

24. Berger S, Schad T, von Wyl V, Ehlert U, Zellweger C, Furrer $\mathrm{H}$, et al. Effects of cognitive behavioral stress management on HIV-1 RNA, CD4 cell counts and psychosocial parameters of HIV-infected persons. AIDS. 2008;22:767-75.

25. Blank MB, Hennessy M, Eisenberg MM. Increasing quality of life and reducing HIV burden: the PATH+ intervention. AIDS Behav. 2014;18:716-25

26. Bormann JE, Gifford AL, Shively M, Smith TL, Redwine L, Kelly A, et al. Effects of spiritual mantram repetition on HIV outcomes: a randomized controlled trial. J Behav Med. 2006;29:359-76.

27. Brown J, Hanson JE, Schmotzer B, Webel AR. Spirituality and optimism: a holistic approach to component-based, self-management treatment for HIV. J Relig Health. 2014;53:1317-28.

28. Cade W, Reeds DN, Mondy KE, Overton E, Grassino J, Tucker S, et al. Yoga lifestyle intervention reduces blood pressure in HIV-infected adults with cardiovascular disease risk factors. HIV Med. 2010;11:379-88.

29. Chhatre S, Metzger DS, Frank I, Boyer J, Thompson E, Nidich S, et al. Effects of behavioral stress reduction Transcendental Meditation intervention in persons with HIV. AIDS Care. 2013;25:1291-7.

30. Duncan LG, Moskowitz JT, Neilands TB, Dilworth SE, Hecht FM, Johnson MO. Mindfulness-based stress reduction for HIV treatment side effects: a randomized, wait-list controlled trial. J Pain Symptom Manag. 2012;43:161-71.

31. Eller LS, Kirksey KM, Nicholas PK, Corless IB, Holzemer WL, Wantland DJ, et al. A randomized controlled trial of an HIV/AIDS Symptom Management Manual for depressive symptoms. AIDS Care. 2013;25:391-9.

32. Fillipas S, Oldmeadow LB, Bailey MJ, Cherry CL. A six-month, supervised, aerobic and resistance exercise program improves self-efficacy in people with human immunodeficiency virus: a randomised controlled trial. Aus J Physiother. 2006;52:185-90.

33. Galantino ML, Shepard K, Krafft L, Laperriere A, Ducette J, Sorbello A, et al. The effect of group aerobic exercise and t'ai chi on functional outcomes and quality of life for persons living with acquired immunodeficiency syndrome. J Altern Complement Med. 2005;11:1085-92.

34. Gayner B, Esplen MJ, DeRoche P, Wong J, Bishop S, Kavanagh L, et al. A randomized controlled trial of mindfulness-based stress reduction to manage affective symptoms and improve quality of life in gay men living with HIV. J Behav Med. 2012;35:272-85.

35. Goujard C, Bernard N, Sohier N, Peyramond D, Lançon F, Chwalow J, et al. Impact of a patient education program on adherence to HIV medication. J Acquir Immune Defic Syndr. 2003;34:191-4.

36. Jones DL, McPherson-Baker S, Lydston D, Camille J, Brondolo E, Tobin JN, et al Efficacy of a group medication adherence intervention among HIV positive women: the SMART/EST Women's Project. AIDS Behav. 2007;11:79-86.

37. Lechner SC, Antoni MH, Lydston D, LaPerriere A, Ishii M, Devieux J, et al. Cognitive-behavioral interventions improve quality of life in women with AIDS. J Psychosom Res. 2003:54:253-61.

38. Li L, Lee S-J, Jiraphongsa C, Khumtong S, lamsirithaworn S, Thammawijaya $P$, et al. Improving the health and mental health of people living with HIV/ AIDS: 12-month assessment of a behavioral intervention in Thailand. Am J Public Health. 2010;100:2418-25.

39. Maharaj SS, Chetty V. Rehabilitation program for the quality of life for individuals on highly active antiretroviral therapy in KwaZulu-Natal, South Africa: a short report. Int J Rehabil Res. 2011;34:360-5.

40. McCain NL, Munjas BA, Munro CL, Elswick R, Robins JLW, Ferreira-Gonzalez A, et al. Effects of stress management on PNI-based outcomes in persons with HIV disease. Res Nurs Health. 2003;26:102-17.

41. Miles MS, Holditch-Davis D, Eron J, Black BP, Pedersen C, Harris DA. An HIV self-care symptom management intervention for African American mothers. Nurs Res. 2003;52:350-60.

42. Molassiotis A, Callaghan P, Twinn S, Lam S, Chung W, Li C. A pilot study of the effects of cognitive-behavioral group therapy and peer support counseling in decreasing psychologic distress and improving quality of life in Chinese patients with symptomatic HIV disease. AIDS Patient Care STDs. 2002;16:83-96

43. Mutimura E, Stewart A, Crowther NJ, Yarasheski KE, Cade WT. The effects of exercise training on quality of life in HAART-treated HIV-positive Rwandan subjects with body fat redistribution. Qual Life Res. 2008;17:377-85.

44. Ogalha C, Luz E, Sampaio E, Souza R, Zarife A, Neto MG, et al. A randomized, clinical trial to evaluate the impact of regular physical activity on the quality of life, body morphology and metabolic parameters of patients with AIDS in Salvador, Brazil. J Acquir Immune Defic Syndr. 2011;57:S179-85.

45. Proeschold-Bell RJ, Belden CM, Parnell H, Cohen S, Cromwell M, Lombard F. A randomized controlled trial of health information exchange between human immunodeficiency virus institutions. J Public Health Manage Pract. 2010;16:521-8.

46. Ruiz I, Olry A, López MÁ, Prada JL, Causse M. Prospective, randomized, two-arm controlled study to evaluate two interventions to improve adherence to antiretroviral therapy in Spain. Enferm Infecc Microbiol Clin. 2010;28:409-15.

47. Sikkema KJ, Hansen NB, Meade CS, Kochman A, Lee RS. Improvements in health-related quality of life following a group intervention for coping with AIDS-bereavement among HIV-infected men and women. Qual Life Res. 2005;14:991-1005

48. Van Tam V, Larsson M, Pharris A, Diedrichs B, Nguyen HP, Nguyen CTK, et al. Peer support and improved quality of life among persons living with HIV on antiretroviral treatment: a randomised controlled trial from north-eastern Vietnam. Health Qual Life Outcomes. 2012;10:1.

49. Wang H, Zhou J, Huang L, Li X, Fennie KP, Williams AB. Effects of nursedelivered home visits combined with telephone calls on medication adherence and quality of life in HIV-infected heroin users in Hunan of China. J Clin Nurs. 2010;19:380-8.

50. Webel AR. Testing a peer-based symptom management intervention for women living with HIV/AIDS. AIDS Care. 2010;22:1029-40.

51. Wu AW, Snyder CF, Huang I-C, Skolasky Jr R, McGruder HF, Celano SA, et al. A randomized trial of the impact of a programmable medication reminder device on quality of life in patients with AIDS. AIDS Patient Care STDs. 2006; 20:773-81. 